\title{
THE TASK OF EARTHQUAKE RESISTANT DESIGN
}

\section{A DIVERGENT PROBLEM}

\author{
R. J. P. Garden*
}

\section{Synopsis}

In most engineering fields, the designer would not feel himself competent if the unresolved uncertainties were as great as those that he has to accept in seismic design problems. Confidence has been somewhat shaken by the record of damage and catastrophe of the last ten years, especially by the uncomfortable fact that the mechanisms of movement which have caused the damage have varied so greatly; there is no sign that nature has exhausted her repertoire。 It is not surprising that studies published in recent years contain a high proportion whose findings reflect the thought "things are worse than we thought". Some examples of such studies are referred to, and ideas expressed as to our future needs.

\section{Introduction}

As solution from first principles is seldom practicable, designers must make use of codes. The rules for static analysis put out from time to time by such bodies as SEAOC, based upon a dynamic approach, have brought noteworthy advances within the scope of relatively simple arithmetic. Nevertheless, the best that Rinne(1) can say about codes is "... an ably administered code is better than no code at all .... not fully accomplishing their objectives". He also said that a practical problem exists in the need to take all the wonderful information presently available and convert it into design criteria for the designer.

Within the range of elastic behaviour, many investigations, such as those reported by Shepherd(2) in this country for instance, can claim useful agreement between the results of dynamic analysis and observed responses of the same buildings when mildly excited. Correlation by many investigators between computed and measured responses have yielded the solid basis for the construction of code rules and for revealing how much "scatter" is to be expected from the rough average values given in codes. Let there be no deception, the "scatter" is very great, even for elastic behaviour of the structure; but in the post elastic range the dispersions resemble the active dissociation of an expanding universe. Such a figure of speech requires some documented justification, and the next section gives references to recent papers which illustrate that there are many factors whose quantitative values need much adhoc labour for reliable determination, and for which the codes and other generalised procedures are not good enough.

* Consulting Engineer, Dunedin.
Uncertainties In The Data

\section{Ground Motions}

The ground motions may be magnified many times by the surface layers of the crust or on the other hand $(3)$ these layers may reduce the response. Damping from the ground and the substructure is said to be of greater help than the amount of damping in the upper structure (4). Housner(1) said in 1965 that the chief engineering problem now is the manner in which soil behaves during an earthquake. Some overdue attention is being paid to different effects that are imposed by different types of seisms $(3,5,6,7,8)$. It now appears that the apparent similarity in spectral qualities is not so readily accepted as evidence that useful generalisation is available for determining the response of actual structures. In the post elastic region, such evidence has always been less convincing. It may be that as we weed out the unfavourable design features, our actual structures will be found to become members of the set for which the generalisations are true. That would be progress indeed.

\section{Response of Structures}

There is advocacy by Nigan and Housner of the need to take into simultaneous account the three axial components of motion recorded by the seismometer(9). There is recognition that motions such as were experienced at Agadir, skopje(10) and on White's Creek in New Zealand should be expected to result in building damage different from that to be expected from the "standard" Californian earthquake. Rosenblueth and Elorduy(11) because they deal with linear systems, are able to build upon past knowledge with less disconformity, but they decide that old methods need revision and find that torsion and overturning have been inadequately catered for.

\section{Torsion}

The vagaries of torsion $(11,12)$ have been further explored with some impressive findings but there remains, no doubt, sufficient unexplored territory to occupy a complete Faculty for some years. There is the admitted but not rationalised occurrence of torsional ground motion, with its polyandrous inheritance from every outstanding feature of rigidity and mass in the neighbourhood. Studies indicate that even for symmetrical structures we should use nominal eccentricities greater than required by present codes. It may be a little disheartening to hear that even our cure-all, plastic yield, can fail us in the matter of torsion, but it is not surprising really, that the loss of restoring force which generally 
accompanies the post elastic state, should fail to save us against torsion whenever such plastic yield occurs prominently in the forner columns and other peripheral supports $(12)$. The need to maintain a restoring force right

through the post elastic range is of importance also in cases of torsion-free displacement, in order to limit permanent set and to avoid early collapse under gravity effects $(4)$.

\section{Uncommon Shapes Of Structure}

Structures of unusual configuration $(13,14)$ can not be adequately designed by code rules, and Blume again, in ref. (15), adds to the advices previously available as to the ways in which we can treat buildings which have such a lack of stiffness in their horizontal members as to remove them from the traditional framed style。

\section{Disparities In Responses}

All the above references, along with ref. (16) serve on us warnings of our inadequacies but also give much advice upon which better rules can be built. For the present writer, the one general feature which stands prominently above all others, is the high probability that a traditional approximate design will prove to be inappropriate when it is tested by the first kind of earthquake severe enough to take the structure well into the plastic range.

Guru and Heidebrecht ${ }^{(15)}$ give interesting attention to the separate influences of a large number of system parameters and conclude with the words ". ... very important to be aware of all the factors that influence response and that simple design and analysis methods which neglect some of these factors can be in gross error in predicting response"; design engineers should not fail to read that paper. of all the causes contributing to this condition, the one respecting which we engineers seem most cupable, lies in the strange fact that we choose to erect structures which, in most cases have response characteristics which result in building oscillations of greater magnitude than the input from the ground motions.

\section{Advances In The Technology}

Many have been active in seeking counter measures and only a defeatist would consider the condition inevitable. No attempt is made here to list famous contributors from the past apart from some further references to papers from Volumes I and II of the Fourth World Conference on Earthquake Engineering to indicate some present trends.

\section{Seismicity From The Records}

Seismic risks in the U.S.A. based on past records, are systematically studied $(17,18)$. As released strain energy can be divided into the part that does work against gravity, the part expended as heat in the epicentral region of crustal dislocation, and the part that radiates as vibrations to regions beyond the epicentral region, the present writer has the impression that most of our seismometer records are derived from the "part that got away". It is relevant to realise that past records show that the first two parts, uplift against gravity, and friction energy on the fault plane, can each demand as much energy as is conventionally assigned to the vibrations of a great magnitude earthquake. Can we infer from (17) that Housner finds that Californian earthquakes show a regular relation between energy absorbed at a fault and energy detected by observatories at a distance?

\section{Prognosis For Seismic Attacks}

Papers in Session A. 1 give consideration to what is able and what is not able to be conjectured about ground motions we have to expect. It appears that inelastic systems are more sensitive to the specific characteristics of the incident motion, so that, with greater ductility factors it becomes more difficult to devise useful generalisations about the attacking seisms, or to rely upon statistical derivations. Studies based upon displacements atc. within areas of severe damage (10) have appeal to the practical engineer.

\section{The Search For More Suitable Structures}

In Sessions $B 3$ and $A 4$, we find stimulating attempts to use new design devices and analyses which make some headqay in the search for self regulating features to limit the unwelcome scatter between the responses which may eventuate out of the many ill-defined contributary factors, which are referred to in the early sections of this paper. Cross bracing is discussed in (22) The important benefit of having a positive slope of that part of the load deflexion curve which is in the postelastic range, is emphasised in (8). Matsushika and Izumi (19) urge that attention be given to limiting the energy accepted into the structure and to limit the strain energy which results. They advocate the development of structures to which the term "aseismic" could for once, be correctly applied. Decrease of earthquake effect through engineering methods is also discussed in (20) and (21). The latter finds that energy absorbers are "not that effective for earthquake type excitations as compared to sine excitations but the force acting on the absorber is very small".

Many actual buildings have in them energy absorbers in the form of live load or loosely attached masses and these may have a major effect upon response. Hanging carcases in the freezing chamber of a coolstore could act both as energy absorbers and as dispersers through damping. Crane loads, chutes, liquids in containers etc. could all make significant contribution. Of still greater general importance are the so-called nonstructural parts of our buildings; the masonry, the timber partitions, the insulations, the service ducts and conduits, the ceiling linings and even the windows have been shown to have a big effect upon response characteristics. The attempts to avoid secondary damage by separating structural from nonstructural elements could be an expensive error of judgement for certain types of structure and certain types of earthquake. The large costs sometimes quoted for repair of secondary damage must be heeded, but when the anticipated seismic threat is from a rare though perhaps severe earthquake, the expense of secondary damage may be thought more accept able than a completely pancaked building. These considerations point towards the argument that we will have large uncertainties until such time as we can separate out the ingredients of the problem. 
Some Favourable Structural Features For Reduction Of Response

\section{The Ingredients}

Various kinds of damping (defined as always involving the loss of energy out of the system), are our best sources of amelioration, but response can also be diminished (or augmented) in the absence of any damping, by choosing particular types of linear elastic mechanisms or of non-linear elastic mechanisms. For instance, energy absorbers, which are linear elastic systems, are used to diminish dynamic response, and would serve this purpose even without extra help from the ever present damping. Non-linear elastic systems which are described as aperiodic systems, will refuse to go into resonance when subjected to periodic inputs of widely ranging periods.

It will be noted that no such class of system as "an inelastic structure" has been mentioned above. Such a system can always be fully described as an equivalent elastic system with added damping effects. One can go further. The dynamic response of any oscillating system subject to any given input can be completely described by determing the response of some arbitary linear elastic system subject to the same given input and subject also to a history of loading such that the loading at any point in time will constrain the arbitrarily chosen system to react for the instant exactly as would the actual system(23). It is tempting to think that this means that the normal mode approach is open for inelastic systems. It is tempting to think that one could, when using any actual earthquake for input, chose a roughly appropriate elastic structure and then determine a time-related counter-input such as would keep the response within acceptable bounds.

The point being emphasised is that the ingredients should be separately recognised. One is suspicious of hearing that a study is inclusive of hysteresis loss but no viscous damping. Is this conducive to clear thinking? Ductility is too often spoken of as though it were a single item requiring no description beyond a single numerical value. All too commonly, authoritative people have said that plastic hinging at a soft storey would mean the absorption at those hinges of excess strain energy from the whole building, whereas the softening at the hinges generally means that the whole structure is spared the need to accept much strain energy.

The benefit of having structures with long natural periods is well known and has been exploited in the spectacular high-rise buildings of the great cities. A new application for limiting the response of a tall structure has been used in the $850^{\circ}$ tall chimney of the Drax Power Station in Yorkshire. The monolithic shell encloses three flues in parallel which are in separated 72 feet lengths supported on rubber mountings. It is reported that this device will reduce the lateral movements at the chimney top to one-fifth or less of the "undamped" value. The research scientists of N.A.S.A. are reported to have found substantial reduction of oscillation to result from a freely suspended chain inside a hollow antenna. How much of the benefit is derived from energy dispersal and how much from the evasion of energy intake?

\section{Conclusion}

In seeking means of limiting tho magnification of response which exists unsought in so many of our buildings, there are a few characteristics which would be helpful, if they are attainable. We would like to be able to call upon strong frictional damping at the small initial stages of displacement. We would like to allow our structures to accept considerable displacement with respect to their foundations without feeding into the structural members any more elastic or inelastic energy than necessary. We would like to have damping of amount appropriate to the energy flow and would like this damping to cause the minimum practical amount of structural degradation or other damage. If the system is to suffer post elastic strain, we will want to have a sufficiency of structural material remaining within its elastic range to ensure that we maintain a restoring force of appropriate magnitude to enable us to deal with permanent set and to avoid what is known as gravity collapse. We do not want buildings which are rapidly whipped up into strong excitation and then asked to deal with the self-inflicted energy by inordinate amounts of plastic yield.

For solving problems of this kind, in the post elastic range of action, the role to be played by testing of physical models will be restricted mainly to small portions of structural frames. Member and material charac. teristics determined as thoroughly as possible by this means will then have to be used in mathematical models for computer solution. Much will depend upon the sagacity of those responsible for the mathematical analysis. Even with pencil-and-paper arithmetic we have al ways been exposed to the risk of being unduly influenced by the narcissistic feed-back from the alpha-numerical patterns which we have ourselves originated. It is with pained resignation that a practical engineer has to recognise the pre-eminence of the computer in this matter, but there is al so some pride that, in this small country, a solid beginning has been made in the exercise of these mysteries.

\section{References}

(1) Symposium, Earthquake Engineering, Vancouver 1965.

(2) Shepherd. 4 WEEC, A3 p.99.

(3) Sexton, Feibush and Keith. 4 WEEC, A4 p. 113 .

(4) Odaka, Susuki and Kinoshita. 4 WEEC, A4 p. 105.

(5) Guru and Heidebrecht. 4 WEEC, A4 p. 1.

(6) Blume and Jhaveri. 4 WEEC, A3 p. 155.

(7) Husid. 4 WEEC, A 4 p. 31.

(8) Iwan. 4 WEEC, A 4 p. 45.

(9) Nigam and Housner. 4 WEEC, A 4 p. 89.

(10) Poceski. 4 WEEC, A2 p. 109.

(11) Rosenblueth and E1orduy. 4 WEEC, Al p.185.

(12) Newmark. 4 WEEC, A3 p. 19.

(13) Penzien. 4 WEEC, A3 p.75.

(14) Newmark. 4 WEEC, B 4 p.37

(15) Blume. 4 WEEC, A3 p. 1.

(16) Jeremiah, 4, v.24 to v.26. 
(17) Housner. 4 WEEC, A1 p. 1.

(18) Algermissen. 4 WEEC, A1 p. 14.

(19) Matsushita and Izumi. 4 WEEC, B3 p.117.

(20) Larios, Salas, Bittner and Silva. 4 WEEC, B3 p. 131 .

(21) Gupta and Chandrasekaran. 4 WEEC, B3 p. 139.

(22) Hanson and Fan. 4 WEEC, A 4 p. 15.

(23) Garden, Shepherd and Sharpe. N.Z. Engineering, Dec. 1970 , p.386.

Errata, Vol 4, No 1

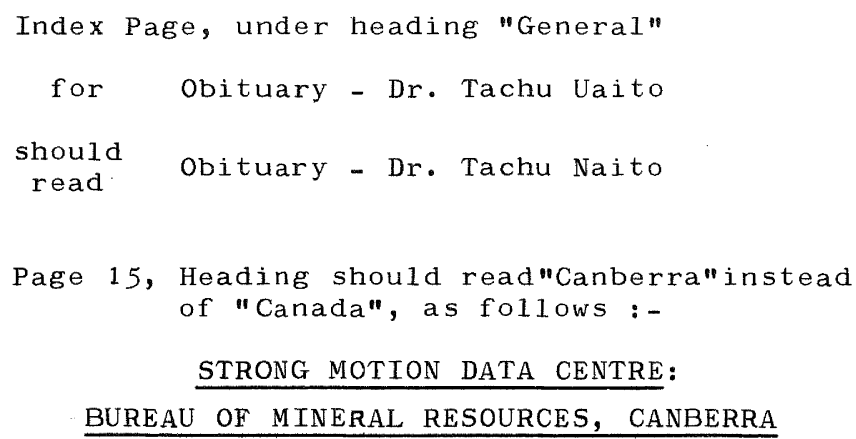

D. Denham* and G. R. Small* 